\title{
BMJ Open Comparative efficacy and safety of anti- HGF/MET pathway agents plus chemotherapy versus chemotherapy alone as first-line treatment in advanced gastric cancer: a protocol for a systematic review and meta-analysis
}

\author{
Zhiyuan Jiang (D) , ${ }^{1}$ Zhaolun Cai (D) , ${ }^{1}$ Qin Ma, ${ }^{1}$ Chaoyong Shen, ${ }^{1}$ Yuan Yin, ${ }^{1}$ \\ Xiaonan Yin, ${ }^{1}$ Chunyu Liu, ${ }^{2,3}$ Chen Chang, ${ }^{4}$ Zhou Zhao, ${ }^{1}$ Mingchun Mu (D) , \\ Bo Zhang (i) 1,5
}

To cite: Jiang Z, Cai Z, Ma Q, et al. Comparative efficacy and safety of anti-HGF/MET pathway agents plus chemotherapy versus chemotherapy alone as first-line treatment in advanced gastric cancer: a protocol for a systematic review and meta-analysis. BMJ Open 2021;11:e049575. doi:10.1136/ bmjopen-2021-049575

- Prepublication history and additional supplemental material for this paper are available online. To view these files, please visit the journal online (http://dx.doi.org/10.1136/ bmjopen-2021-049575).

ZJ, ZC and QM contributed equally.

Received 27 January 2021 Accepted 22 November 2021

Check for updates

(c) Author(s) (or their employer(s)) 2021. Re-use permitted under CC BY-NC. No commercial re-use. See rights and permissions. Published by BMJ.

For numbered affiliations see end of article.

Correspondence to Professor Bo Zhang; hxwcwk@126.com

\section{ABSTRACT}

Introduction Phase $\mathrm{I} / \mathrm{I}$ clinical trials suggested that the hepatocyte growth factor (HGF)/mesenchymal-epithelial transition (MET) pathway-targeted agents were active in suppression of gastric cancer (GC). Randomised controlled trials (RCTs) were undertaken assessing whether the addition of anti-HGF/MET agent (rilotumumab or onartuzumab) to chemotherapy improves survival outcomes of advanced GC, but conflict conclusions were reached. Therefore, we plan to perform this systematic review and meta-analysis to synthesise evidence concerning efficacy and safety of antiHGF/MET agents combined with chemotherapy as the firstline treatment to advanced GC.

Methods and analysis Systematic searches of the PubMed, Embase and the Cochrane Central Register of Controlled Trials will be performed with no language restriction from inception to 31 January 2022 to identify RCTs exploring the comparative efficacy and safety of anti-HGF/MET agents plus chemotherapy as first-line treatment in advanced GC. The primary outcome will be the time-to-event progression-free survival and overall survival, and the secondary outcomes will be disease control rate, overall adverse events rate and grade $3-5$ adverse events rate. Statistical heterogeneity will be assessed by visual inspection of forest plots and measured using the $\mathrm{I}^{2}$ statistics. A fixed-effect model will be used when heterogeneity is low otherwise, a random-effect model will be chosen. Publication bias will be assessed by funnel plots; subgroup analysis and sensitivity analysis will be performed in the right context. For each outcome, we will perform data synthesis using Rev Man V.5.3 software, and compile 'summary of findings' tables using GRADEpro software. Ethics and dissemination There is no requirement for ethics approval because no individual data will be collected in this research. It is anticipated that the dissemination of results will take place at conferences and through publication in a peer-review journal, any adjustments from the protocol will be clearly documented and explained in its final report. PROSPERO registration number CRD42020177404.
Strengths and limitations

- This protocol is written in accordance with the Preferred Reporting Items for Systematic Reviews and Meta-Analysis Protocols (PRISMA-P) guidelines, and we intend to conduct our study according to the PRISMA guidelines.

- Publishing this protocol ensures that we are transparent with the development process we will be using for this study, to reduce the likelihood of duplication as well as minimise the effects of bias on our study.

- Highest level of evidence for informed decisionmaking might be made available from this systematic review because it includes only randomised controlled trials.

- The heterogeneity of chemotherapy regimen and patient baseline characteristics may lead to the degradation of evidence quality.

- This study may also be limited by not many eligible studies and insufficient sample size.

\section{INTRODUCTION}

Gastric cancer (GC) is an important cancer worldwide and is responsible for over 1000000 new cases and an estimated 783000 deaths (equating to 1 in every 12 deaths globally) in 2018, making it the fifth most frequently diagnosed cancer and the third leading cause of cancer death. ${ }^{1}$ Doublet or triplet chemotherapy with a platinum-fluoropyrimidine backbone remains the mainstay of treatment for fit patients with unresectable advanced GC, such as the mFOLFOX6 regimen (fluorouracil, leucovorin calcium and oxaliplatin). ${ }^{23}$ Other effective therapeutics for GC in the refractory setting include taxanes, irinotecan hydrochloride and 
the vascular endothelial growth factor receptor-2 inhibitor ramucirumab (in the second-line setting) ${ }^{4-6}$ In addition, chemotherapy plus trastuzumab is standard of care for patients with human epidermal growth factor receptor (EGFR) 2 positive advanced GC. ${ }^{7}$ Also, the addition of nivolumab to first-line chemotherapy in gastric and gastrooesophageal junction adenocarcinoma improved survival, progression-free survival (PFS), response and findings that led to regulatory approval in some countries. ${ }^{8}$ Despite these efforts, prognosis for unresectable advanced GC remains dismal, with reported median overall survival (OS) times inferior to 1 year. $^{910}$

Other targeted therapies have been developed to counteract the deregulation of signal transduction pathways, including EGFR and hepatocyte growth factor (HGF)/ mesenchymal-epithelial transition (MET) pathways. ${ }^{11} 12$ In advanced GC, EGFR overexpression occurs in up to $50 \%$ of patients. ${ }^{13}$ Similarly, abnormal HGF and MET upregulation occurs in GC, with MET overexpression in 18\%-82\% of patients. ${ }^{14}$ The HGF receptor that activates key oncogenic pathways through RAS, PI3K and STAT3 plays an important role in tumourigenesis and is encoded by MET oncogene. ${ }^{15}$ Signalling through the HGF/MET pathway stimulates tissue repair and regeneration in normal tissue but also can promote proliferation, survival and metastasis in tumour cells. ${ }^{16}$ In GC, dysregulation of the MET/HGF pathway is associated with poor prognosis and more aggressive disease, with MET activation stimulating tumour invasiveness. $^{17} 18$

Rilotumumab, a monoclonal antibody targeting HGF, was shown tolerable and active in various advanced solid tumours. ${ }^{190}$ Onartuzumab is a recombinant, fully humanised, monovalent monoclonal antibody, and it prevents MET from binding with HGF and restricts cellular signalling via the MET pathway by binding with the extracellular domain of MET. Preliminary data from phase I/ II studies indicated that HGF/MET-targeted agents, including rilotumumab and onartuzumab, are active in GC. $^{21}{ }^{22}$ In addition, several randomised controlled trials (RCTs) have been conducted to assess whether the addition of these anti-HGF/MET agents to chemotherapy as first-line therapy for advanced GC improves efficacy outcomes when compared with chemotherapy alone, but obtained some conflict results. ${ }^{923-25}$

Therefore, we plan to perform this systematic review and meta-analysis, to first synthesise evidence concerning efficacy and safety of anti-HGF/MET pathway agents combined with chemotherapy as the first-line treatment in advanced GC. The protocol for our research is reported according to the Preferred Reporting Items for Systematic Review and Meta-Analysis Protocols (PRISMA-P), ${ }^{26} 27$ its PRISMA-P checklist file is attached (online supplemental file 1). The findings of our research will be published in a peer-reviewed journal, also in the event of insignificant results, and thereby it will be disseminated to clinicians and public available.

\section{OBJECTIVES}

The goal of this systematic review is to explore the questions as follows:

1. Whether the anti-HGF/MET agents plus chemotherapy are more effective than chemotherapy alone as firstline treatment to advanced GC.

2. And how safe is the combination regimen compared with chemotherapy alone?

\section{METHODS AND ANALYSIS}

This systematic review and meta-analysis will be conducted according to the PRISMA guidelines. ${ }^{28}$

\section{Criteria for considering studies \\ Study characteristics}

This systematic review will include only RCTs comparing the efficacy and safety of anti-HGF/MET agents plus chemotherapy versus chemotherapy alone as firstline treatment in advanced GC. Studies should have a minimum of 24-month follow-up. Duplicates and full text unavailable due to specific reasons will be excluded.

\section{Population}

Patients diagnosed as advanced GC and had not received any chemotherapy or immunotherapy previously will be included.

\section{Intervention}

The intervention will be anti-HGF/MET agent (rilotumumab or onartuzumab) plus chemotherapy.

\section{Comparator}

The comparator will be chemotherapy plus placebo or chemotherapy alone.

\section{Outcomes}

Primary outcome: time-to-event PFS and OS, or relevant data to estimate them.

Secondary outcome: disease control rate, overall adverse events rate and grade $3-5$ adverse events rate, or relevant data to estimate them.

\section{Search strategy}

The search strategy will be conducted in two stages.

1. Bibliographic database searches: a systematic search of PubMed, Embase and the Cochrane Central Register of Controlled Trials will be performed with no restrictions on language from inception to 31 January 2022 to identify all relevant studies. The details of search strategy and syntax are shown in online supplemental file 2.

2. Searching for other sources: the references of relevant articles will be manually searched to further identify eligible studies, and their full texts will be retrieved.

\section{Study selection and data extraction}

Records identified according to search strategy will be exported to EndNote V.X8 software. Three reviewers (ZJ, $\mathrm{ZC}$ and $\mathrm{CS}$ ) will then independently screen their titles 
and abstracts. Each eligible title/abstract will require two votes. Subsequently, these reviewers will reassess the full texts of the identified studies, verifying the reasons for inclusion and exclusion.

Data extraction for included studies will be conducted by two reviewers (ZJ and QM) independently using a standardised electronic data extraction form (table 1). The following data will be extracted from all the included studies: first author, publication year, study design, study period, country(region), female rate, age (median and range), follow-up (median and range), chemotherapy regimen, anti-HGF/MET agent, sample size, Eastern Cooperative Oncology Group (ECOG) performance status, tumour site, tumour stage, histologic grade, disease control rate, overall adverse events rate and grade 3-5 adverse events rate, and survival outcomes. If multiple HRs for a survival outcome are presented in a paper, we will choose the one adjusted for the greatest number of confounders. ${ }^{29}$ All disputes in the process of study selection and data extraction will be resolved through team negotiation.

\section{Risk of bias assessment}

Two reviewers (ZC and $\mathrm{QM}$ ) will assess the risk of bias of included studies independently using the Cochrane Collaboration's risk of bias tool. ${ }^{30}$ Disagreements will be resolved by discussion, where necessary, in consultation with the third reviewer (YY). Results of this meta-analyses will be interpreted in light of risk of bias assessment of the included studies.

\section{Measurements}

Time-to-event outcome will be analysed by the pooled HR, and the OR will be used to pool dichotomous outcome. Results will be presented as summary relative effect size (HR or OR) with $95 \%$ CI.

\section{Strategy for data synthesis}

We will undertake this meta-analysis according to the recommendations stated in the Cochrane Handbook for Systematic Reviews of Interventions. ${ }^{31}$ The extracted data will be imported into Rev Man V.5.3 software (Copenhagen: The Nordic Cochrane Centre, The Cochrane Collaboration, 2014) by the first reviewer and checked by the second one. The HRs of PFS as well as OS comparing the two interventions will be pooled in the meta-analysis. As for disease control rate, overall adverse events rate, and grade $3-5$ adverse events rate, we will calculate the pooled ORs. Statistical heterogeneity will be assessed by visual inspection of forest plots and measured using the $\mathrm{I}^{2}$ statistics. $\mathrm{I}^{2}<50 \%$ or $\geq 50 \%$ indicates low or high heterogeneity, respectively. A fixed-effect model will be used when heterogeneity is low; otherwise, a random-effect model will be chosen. When substantial heterogeneity is detected, subgroup analysis and sensitivity analysis will be performed to investigate its possible sources. If quantitative synthesis is not possible due to heterogeneity, we will perform a narrative synthesis.
Table 1 Data extraction form

\begin{tabular}{|c|c|}
\hline Study details & \\
\hline General information & \\
\hline First author & \\
\hline Year of publication & \\
\hline D Region & \\
\hline Study period & \\
\hline Study eligibility & \\
\hline Study design & \\
\hline - Follow-up (median and range: month) & \\
\hline Dopulation & \\
\hline - Intervention & \\
\hline Comparator & \\
\hline - Outcome diagnostic criteria & \\
\hline - Confounding variables & \\
\hline Include or exclude & Include $\square$ Exclude $\square$ \\
\hline Reason(s) for exclusion & \\
\hline Characteristics of included studies & \\
\hline Sample size & $\begin{array}{l}\text { Combination therapy group } \\
\text { Chemotherapy group }\end{array}$ \\
\hline Data source & \\
\hline Age (median and range) & \\
\hline - Gender (female rate) & \\
\hline - Tumour size $(\mathrm{cm})$ & \\
\hline Tumour site & \\
\hline - Histologic grade & \\
\hline - TNM stage & \\
\hline ECOG performance status & \\
\hline - Chemotherapy regimen & \\
\hline - Anti-HGF/MET agent & \\
\hline D Subgroups & \\
\hline - Key conclusion(s) & \\
\hline Primary outcomes & \\
\hline $\begin{array}{l}\text { HRs (comparing combination therapy } \\
\text { and chemotherapy group) with } 95 \% \mathrm{Cl}\end{array}$ & \\
\hline OS & \\
\hline PFS & \\
\hline - Relevant data to calculate HRs & \\
\hline os & \\
\hline PFS & \\
\hline
\end{tabular}

Other data

Second outcomes

Disease control rate

Combination therapy group Chemotherapy group

Overall adverse events rate

Combination therapy group Chemotherapy group

Grade 3-5 adverse events rate

Combination therapy group Chemotherapy group

Other data

Combination therapy group Chemotherapy group

ECOG, Eastern Cooperative Oncology Group; HGF, hepatocyte growth factor; MET, mesenchymal-epithelial transition; OS, overall survival; PFS, progression-free survival; TNM, tumour, node, metastases. 


\section{Dealing with missing data}

When an HR of PFS or OS and its upper or lower limit of $95 \%$ CI are provided, we can calculate its lnHR (the natural logarithm of HR) and SE, and then merge HRs. When the above data are incomplete, we will attempt to contact the authors to retrieve it. If we do not get an effective response in 2 weeks, we will try to estimate some or all of the lnHR, the logrank observed minus expected events $(\mathrm{O}-\mathrm{E})$, the logrank variance and the variance of the lnHR by indirect methods. ${ }^{32}$ If even these indirect methods cannot be applied, we will consider to generate the necessary statistics from published Kaplan-Meier curves. ${ }^{32}$ To pool ORs of disease control rate, overall adverse events rate, or grade 3-5 adverse events rate, we will record data on the total number of participants and the incidence of events in each arm of each study.

When a study fails to provide necessary statistics by all mentioned methods, we will contact the authors to extract details, and studies failing to provide these necessary data will be excluded from meta-analysis.

\section{Sensitivity analysis and subgroup analyses}

We will apply the leave-one-out sensitivity analysis to evaluate the robustness of the results.

Subgroup analyses are planned as follows:

1. Sex: male versus female

2. Primary tumour site: stomach versus oesophagogastric junction

3. ECOG status: 0 versus $\geq 1$

4. Prior gastrectomy or oesophagectomy: with versus without.

5. Tumour biomarker status: MET positive versus MET negative.

6. Agent: anti-HGF agent (rilotumumab) versus antiMET agent (onartuzumab).

\section{Publication bias assessment}

Detecting and overcoming publication bias are problematic and firm guidance is not yet offered; thus, we will use visual inspection of funnel plots to assess publication bias, with results being interpreted cautiously. ${ }^{33} 34$

\section{Quality of the evidence}

The overall quality of evidence for each outcome will be assessed using the five Grading of Recommendations, Assessment, Development and Evaluations approach. The direct evidence from RCTs begins at high quality, and the overall quality will be analysed on five downgrade considerations (study limitations, consistency of effect, imprecision, indirectness and publication bias) and three up-grade considerations (large magnitude of effect, dose-response relation and plausible confounders or biases), and finally rate it as high, moderate, low or very low. ${ }^{35-42}$ We will provide all decisions to down-grade or up-grade the quality of studies with clear arguments in footnotes to aid the reader's understanding of the process.

\section{Presentation and reporting of results}

We will follow the PRISMA statement to report our findings. ${ }^{28}$ The study selection process will be summarised by a PRISMA flow chart (online supplemental file 3). The characteristics of each enrolled study will be tabulated in detail. The forest plots generated by Rev Man V.5.3 software will be used to present the pooled estimates. For each outcome, 'summary of findings' table will be compiled by GRADEpro software (GRADEpro GDT 2015).

\section{Patient and public involvement}

Patients and the public will not be involved in the design, or conduct, or reporting, or dissemination plans of this research because it will be based on published studies without collecting raw individual data.

\section{Ethics and dissemination}

There is no ethics approval required for this systematic review due to no patient data being collected at an individual level. We will seek to present the findings at relevant conferences and publish in an influential open access journal, any deviations from the protocol will be clearly explained in its final report.

\section{Author affiliations}

${ }^{1}$ Department of Gastrointestinal Surgery, Sichuan University West China Hospital, Chengdu, China

${ }^{2}$ Evidence-Based Pharmacy Center, Sichuan University West China Second University Hospital, Chengdu, China

${ }^{3}$ Key Laboratory of Birth Defects and Related Diseases of Women and Children,

Sichuan University, Chengdu, China

${ }^{4}$ Department of Abdominal Oncology, Cancer Center, Sichuan University West China Hospital, Chengdu, China

${ }^{5}$ Sanya People's Hospital/ West China (Sanya) hospital, Sanya, China

Contributors ZJ, ZC and QM contributed equally to the research and designed the protocol and drafted the manuscript. The original idea of this research was conceived by BZ and ZJ. ZJ, ZC, ZZ, MM and XY participated in developing the eligibility criteria, search strategy, data extraction methods and data summary plan. ZJ, ZC, CC, CL and CS will search for studies and extract and analyse the data. BZ and $Y Y$ will supervise this work.

Funding This study was funded by the Construction Project of Hainan Province Clinical Medical Center, 1.3.5 project for disciplines of excellence, West China Hospital, Sichuan University (number: ZYJC18034) and 1.3.5 project for disciplines of excellence-Clinical Research Incubation Project, West China Hospital, Sichuan University (number: 19HXFH054).

Competing interests None declared.

Patient consent for publication Not applicable.

Ethics approval This study does not involve human participants.

Provenance and peer review Not commissioned; externally peer reviewed. No data are available.

Supplemental material This content has been supplied by the author(s). It has not been vetted by BMJ Publishing Group Limited (BMJ) and may not have been peer-reviewed. Any opinions or recommendations discussed are solely those of the author(s) and are not endorsed by BMJ. BMJ disclaims all liability and responsibility arising from any reliance placed on the content. Where the content includes any translated material, BMJ does not warrant the accuracy and reliability of the translations (including but not limited to local regulations, clinical guidelines, terminology, drug names and drug dosages), and is not responsible for any error and/or omissions arising from translation and adaptation or otherwise. 
Open access This is an open access article distributed in accordance with the Creative Commons Attribution Non Commercial (CC BY-NC 4.0) license, which permits others to distribute, remix, adapt, build upon this work non-commercially, and license their derivative works on different terms, provided the original work is properly cited, appropriate credit is given, any changes made indicated, and the use is non-commercial. See: http://creativecommons.org/licenses/by-nc/4.0/.

\section{ORCID iDs}

Zhiyuan Jiang http://orcid.org/0000-0003-2483-3413

Zhaolun Cai http://orcid.org/0000-0002-3706-6703

Mingchun Mu http://orcid.org/0000-0003-0133-8882

Bo Zhang http://orcid.org/0000-0002-0254-5843

\section{REFERENCES}

1 Bray F, Ferlay J, Soerjomataram I, et al. Global cancer statistics 2018: GLOBOCAN estimates of incidence and mortality worldwide for 36 cancers in 185 countries. CA Cancer J Clin 2018;68:394-424.

2 Smyth EC, Verheij M, Allum W, et al. Gastric cancer: ESMO clinical practice guidelines for diagnosis, treatment and follow-up. Ann Oncol 2016;27:v38-49.

3 Shah MA. Update on metastatic gastric and esophageal cancers. J Clin Oncol 2015;33:1760-9.

4 Kang JH, Lee SI, Lim DH, et al. Salvage chemotherapy for pretreated gastric cancer: a randomized phase III trial comparing chemotherapy plus best supportive care with best supportive care alone. J Clin Oncol 2012;30:1513-8.

5 Ford HER, Marshall A, Bridgewater JA, et al. Docetaxel versus active symptom control for refractory oesophagogastric adenocarcinoma (COUGAR-02): an open-label, phase 3 randomised controlled trial. Lancet Oncol 2014;15:78-86.

6 Fuchs CS, Tomasek J, Yong CJ, et al. Ramucirumab monotherapy for previously treated advanced gastric or gastro-oesophageal junction adenocarcinoma (regard): an international, randomised, multicentre, placebo-controlled, phase 3 trial. Lancet 2014;383:31-9.

7 Bang Y-J, Van Cutsem E, Feyereislova A, et al. Trastuzumab in combination with chemotherapy versus chemotherapy alone for treatment of HER2-positive advanced gastric or gastro-oesophageal junction cancer (ToGA): a phase 3, open-label, randomised controlled trial. Lancet 2010;376:687-97.

8 Ilson DH. Immunotherapy in esophagogastric cancer. Clin Adv Hematol Oncol 2021;19:639-47.

9 Shah MA, Cho J-Y, Tan IB, et al. A randomized phase II study of FOLFOX with or without the Met inhibitor Onartuzumab in advanced adenocarcinoma of the stomach and gastroesophageal junction. Oncologist 2016;21:1085-90.

10 Lordick F, Kang Y-K, Chung H-C, et al. Capecitabine and cisplatin with or without cetuximab for patients with previously untreated advanced gastric cancer (expand): a randomised, open-label phase 3 trial. Lancet Oncol 2013;14:490-9.

11 Nicholson RI, Gee JMW, Harper ME. Egfr and cancer prognosis. Eur $J$ Cancer 2001;37:9-15.

12 Gherardi E, Birchmeier W, Birchmeier C, et al. Targeting Met in cancer: rationale and progress. Nat Rev Cancer 2012;12:89-103.

13 Kasper S, Schuler M. Targeted therapies in gastroesophageal cancer. Eur J Cancer 2014:50:1247-58.

14 Yu S, Yu Y, Zhao N, et al. C-Met as a prognostic marker in gastric cancer: a systematic review and meta-analysis. PLoS One 2013;8:e79137.

15 Liu X, Yao W, Newton RC, et al. Targeting the c-Met signaling pathway for cancer therapy. Expert Opin Investig Drugs 2008;17:997-1011.

16 Blumenschein GR, Mills GB, Gonzalez-Angulo AM. Targeting the hepatocyte growth factor-cMET axis in cancer therapy. J Clin Oncol 2012;30:3287-96.

17 Samamé Pérez-Vargas JC, Biondani P, Maggi C, et al. Role of cMET in the development and progression of colorectal cancer. Int $\mathrm{J} \mathrm{Mol}$ Sci 2013;14:18056-77.

18 Trusolino L, Bertotti A, Comoglio PM. Met signalling: principles and functions in development, organ regeneration and cancer. Nat Rev Mol Cell Biol 2010;11:834-48.

19 Gordon MS, Sweeney CJ, Mendelson DS, et al. Safety, pharmacokinetics, and pharmacodynamics of AMG 102, a fully human hepatocyte growth Factor-Neutralizing monoclonal antibody, in a first-in-human study of patients with advanced solid tumors. Clin Cancer Res 2010;16:699-710.

20 Rosen PJ, Sweeney CJ, Park DJ, et al. A phase lb study of AMG 102 in combination with bevacizumab or motesanib in patients with advanced solid tumors. Clin Cancer Res 2010;16:2677-87.

21 Catenacci DVT, Henderson L, Xiao S-Y, et al. Durable complete response of metastatic gastric cancer with anti-Met therapy followed by resistance at recurrence. Cancer Discov 2011;1:573-9.

22 Iveson T, Donehower RC, Davidenko I, et al. Rilotumumab in combination with epirubicin, cisplatin, and capecitabine as first-line treatment for gastric or oesophagogastric junction adenocarcinoma: an open-label, dose de-escalation phase 1B study and a double-blind, randomised phase 2 study. Lancet Oncol 2014;15:1007-18.

23 Malka D, François E, Penault-Llorca F, et al. Folfox alone or combined with rilotumumab or panitumumab as first-line treatment for patients with advanced gastroesophageal adenocarcinoma (PRODIGE 17-ACCORD 20-MEGA): a randomised, open-label, threearm phase II trial. Eur J Cancer 2019;115:97-106.

24 Shah MA, Bang Y-J, Lordick F, et al. Effect of fluorouracil, leucovorin, and oxaliplatin with or without Onartuzumab in HER2-negative, MET-Positive gastroesophageal adenocarcinoma: the METGastric randomized clinical trial. JAMA Oncol 2017;3:620-7.

25 Catenacci DVT, Tebbutt NC, Davidenko I, et al. Rilotumumab plus epirubicin, cisplatin, and capecitabine as first-line therapy in advanced MET-positive gastric or gastro-oesophageal junction cancer (RILOMET-1): a randomised, double-blind, placebocontrolled, phase 3 trial. Lancet Oncol 2017;18:1467-82.

26 Shamseer L, Moher D, Clarke M, et al. Preferred reporting items for systematic review and meta-analysis protocols (PRISMA-P) 2015 : elaboration and explanation. BMJ 2015;350:g7647.

27 Moher D, Shamseer L, Clarke M, et al. Preferred reporting items for systematic review and meta-analysis protocols (PRISMA-P) 2015 statement. Syst Rev 2015;4:1.

28 Moher D, Liberati A, Tetzlaff J, et al. Preferred reporting items for systematic reviews and meta-analyses: the PRISMA statement. BMJ 2009;339:b2535.

29 Hunink MG, Wong JB. Meta-Analysis of failure-time data with adjustment for covariates. Med Decis Making 1994;14:59-70.

30 Higgins JPT, Altman DG, Gøtzsche PC, et al. The Cochrane Collaboration's tool for assessing risk of bias in randomised trials. BMJ 2011;343:d5928

31 Cumpston M, Li T, Page MJ, et al. Updated guidance for trusted systematic reviews: a new edition of the Cochrane Handbook for systematic reviews of interventions. Cochrane Database Syst Rev 2019;10:Ed000142.

32 Tierney JF, Stewart LA, Ghersi D, et al. Practical methods for incorporating summary time-to-event data into meta-analysis. Trials 2007;8:16.

33 Egger M, Davey Smith G, Schneider M, et al. Bias in meta-analysis detected by a simple, graphical test. BMJ 1997;315:629-34.

34 Li F, Liu G, Roudi R, et al. Do statins improve outcomes for patients with non-small cell lung cancer? A systematic review and metaanalysis protocol. BMJ Open 2018;8:e022161.

35 Balshem H, Helfand M, Schünemann HJ, et al. Grade guidelines: 3. rating the quality of evidence. J Clin Epidemiol 2011;64:401-6.

36 Guyatt GH, Oxman AD, Kunz R, et al. GRADE guidelines 6. Rating the quality of evidence--imprecision. J Clin Epidemiol 2011;64:1283-93.

37 Guyatt GH, Oxman AD, Kunz R, et al. GRADE guidelines: 8. Rating the quality of evidence--indirectness. J Clin Epidemiol 2011;64:1303-10.

38 Guyatt GH, Oxman AD, Kunz R, et al. GRADE guidelines: 7. Rating the quality of evidence--inconsistency. J Clin Epidemiol 2011;64:1294-302.

39 Guyatt GH, Oxman AD, Montori V, et al. GRADE guidelines: 5. Rating the quality of evidence--publication bias. J Clin Epidemiol 2011;64:1277-82.

40 Guyatt $\mathrm{GH}$, Oxman AD, Sultan S, et al. Grade guidelines: 9. rating up the quality of evidence. J Clin Epidemiol 2011;64:1311-6.

41 Guyatt GH, Oxman AD, Vist G, et al. GRADE guidelines: 4. Rating the quality of evidence--study limitations (risk of bias). J Clin Epidemiol 2011;64:407-15

42 Kien C, Gartlehner G, Kaminski-Hartenthaler A, et al. [GRADE guidelines: 9. Rating up the quality of evidence]. Z Evid Fortbild Qual Gesundhwes 2013;107:249-55. 